\title{
3D Convolutional Neural Networks for Diagnosis of Alzheimer's Disease via structural MRI
}

\author{
Ekin Yagis ${ }^{1}$, Luca Citi $^{1}$, Stefano Diciotti ${ }^{2}$, Chiara Marzi $^{2}$, Selamawet Workalemahu Atnafu ${ }^{2}$, and Alba G. Seco De Herrera ${ }^{1}$ \\ ${ }^{1}$ Computer Science and Electrical Engineering (CSEE), University of Essex, Colchester, United Kingdom \\ ${ }^{2}$ Department of Electrical, Electronic, and Information Engineering "Guglielmo Marconi”, University of Bologna, Bologna, Italy
}

\begin{abstract}
Alzheimer's Disease (AD) is a widespread neurodegenerative disease caused by structural changes in the brain and leads to deterioration of cognitive functions. Patients usually experience diagnostic symptoms at later stages after irreversible neural damage occurs. Therefore, early detection of AD is crucial to start treatments to decelerate the progress of the disease and to maximize patients' quality of life. With the rapid advances in machine learning and scanning, early detection of $\mathrm{AD}$ may be possible via computer-assisted systems using neuroimaging data. Among all, deep learning utilizing magnetic resonance imaging (MRI) has become a prominent tool due to its capability to extract high-level features through local connectivity, weight sharing, and spatial invariance. This paper describes our investigation of the classification accuracy based on two publicly available data sets, namely, ADNI and OASIS, by building a 3D VGG variant convolutional network (CNN). We used 3D models to avoid information loss, which occurs during the process of slicing 3D MRI into 2D images and analyzing them by $2 D$ convolutional filters. We also conducted a pre-processing of the data to enhance the effectiveness and classification performance of the model. The proposed model achieved $73.4 \%$ classification accuracy on ADNI and $69.9 \%$ on OASIS dataset with 5-fold cross-validation (CV), outperforming 2D network models.
\end{abstract}

Index Terms-Alzheimer's Disease, Deep Learning, Image Classification, 3D CNN, MRI, Neuroimaging

\section{INTRODUCTION}

Alzheimer's Disease (AD) is the most common type of dementia, which is caused by the deterioration of cognitive and memory functions [1]. Pathologically, AD is characterized by the accumulation of extracellular $\beta$-amyloid $(\mathrm{A} \beta$ ) plaques and cytoplasmic neurofibrillary tangles (NFTs) which have a microtubule-associated protein called tau [2]. In healthy neurons, tau protein normally stabilizes the microtubules [3]. However, abnormal changes in brain chemistry cause tau protein molecules to detach from microtubules and form neurofibrillary tangles destroying the brain cells' ability to communicate with other cells [4].

Some recent studies reveal that AD may begin 20 years or more before any symptoms appear and the disease is clinically diagnosed [5]-[9]. Only after a certain stage, patients may experience diagnostic symptoms such as deterioration in

\footnotetext{
*Data used in preparation of this article were obtained from the Alzheimer's Disease Neuroimaging Initiative (ADNI) database (adni.loni.usc.edu). As such, the investigators within the ADNI contributed to the design and implementation of ADNI and/or provided data but did not participate in analysis or writing of this report. A complete listing of ADNI investigators can be found at: http://adni.loni.usc.edu/wp-content/uploads/howtoapply/ ADNIAcknowledgementList.pdf
}

memory and decline in cognitive abilities when the irreversible neurological damage already occurs. Therefore, early and accurate diagnosis of $\mathrm{AD}$ is crucial and may be possible via computer-assisted analytical techniques. Receiving an early diagnosis of $\mathrm{AD}$ will enable patients to benefit from various treatments, plan their future, and maximize their life quality.

As AD progresses, the structure of the brain undergoes some changes, such as the shrinkage of the cerebral cortex and hippocampus and the expansion of ventricles [10], [11]. Through numerous medical imaging techniques like magnetic resonance imaging (MRI), positron emission tomography (PET) and computed tomography (CT), some of these changes can be detected earlier. Notably, a $T_{1}$-weighted MRI scan of the brain reveals high-resolution structural information of the brain and can be used to identify atrophic changes in the temporal lobes [12].

Throughout the last decade, multiple studies have been focusing on the automatic diagnosis of $\mathrm{AD}$ using different methods [13]-[15]. Among those, deep learning (DL) has come to the fore as one of the most promising tools to address $\mathrm{AD}$ diagnosis and prognosis. In DL models, discriminative features may be extracted automatically from the raw data resulting in end-to-end learning design.

In this work, we propose an end-to-end $\mathrm{AD}$ classifier, which takes $T_{1}$ weighted MRI as input. We implemented a 3D VGG (a deep neural network model implemented by Oxford Visual Geometry Group (VGG)) variant convolutional neural network (CNN) to overcome the limitations regarding the feature extraction from brain MRI and preserve spatial relations. Figure 1 provides an illustration of the network architecture.

The paper is organized as follows: after this introduction, a brief of related work is given in Section II. Section III provides the details of the proposed model, including the dataset and classification algorithm of CNN. Experimental results are presented in Section IV. Finally, Section V concludes the paper with some final remarks.

\section{RELATED WORK}

DL has become a popular and powerful technique with the advance of the computational power of GPU clusters and big data analytics, and as a result, rapidly expanded into various fields. In medical image analysis, several neuroimaging studies have utilized DL models for diagnosis of AD [16]-[21]. 


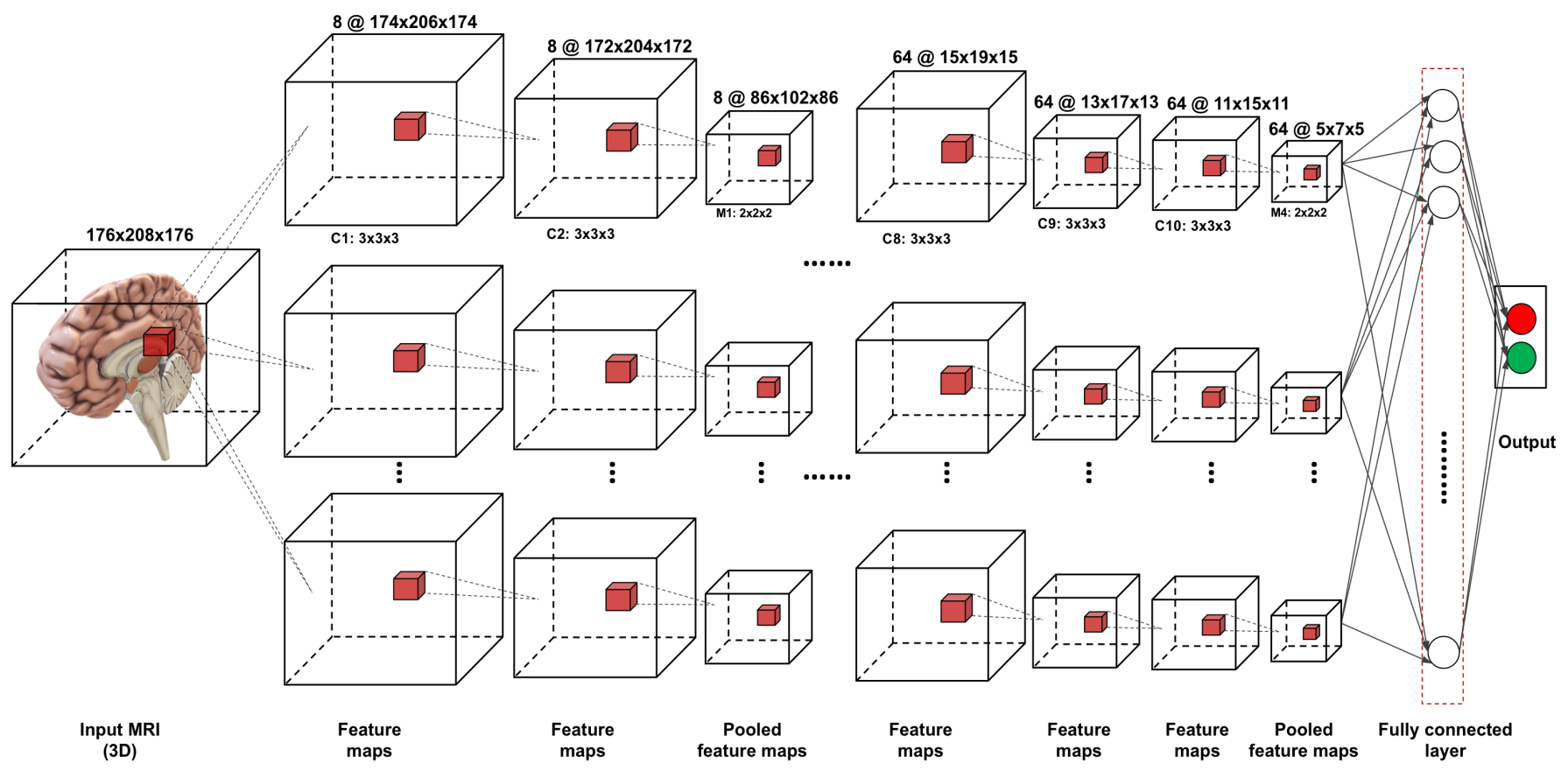

Fig. 1: Overview of the 3D convolutional neural network $(\mathrm{CNN})$ architecture. 3D boxes show input and feature maps.

Various studies used a set of 2D slices extracted from the MRI volume as input to the 2D CNN architectures [22][28]. Farooq et al. [22] used a 2D CNN model for 4-way classification of Alzheimer's into AD, MCI (Mild Cognitive Impairment), LMCI (Late Cognitive Impairment) and HC (Healthy Control) using structural MRI images. Sarraf et al. [29] utilized CNN and the famous architecture LeNet-5 to classify functional MRI data of AD's patients from healthy controls. In [24], Hon et al. used VGG16 and Inception V4 to classify AD using transfer learning. Finally, in 2019, Jain et al. [30] presented the CNN model for the 3-way AD classification. However, in most of these studies, it is not clear if data division was done at the subject-level, calling into question the validity of the results due to potential data leakage [21], [28], [31]. Another possible problem in the 2D approach is the loss of information from 3D MRI when sliced and analyzed by 2D convolutional filters.

Some studies addressed 3D networks to solve the issue of insufficient information in the 2D slice-level approach [16], [17]. Even though these models are computationally more expensive, they have a higher capability to extract discriminative features from three-dimensional MRI data. Korolev et al. [20] used 3D residual neural network architecture together with several regularization techniques for $\mathrm{AD}$ classification. In 2018, Hosseini-Asl et al. [32], utilized a pre-trained 3DAdaptive CNN classifier with used scans from the CADDementia dataset for the classification of AD vs. HC. However, the details regarding CV methodology and classification decisions are not presented in this study. Wang et al. [33] proposed an ensemble of 3D densely connected convolutional networks (3D-DenseNets) for three-class $\mathrm{AD}, \mathrm{MCI}$, and $\mathrm{HC}$ diagnosis. In their model, MRI scans of the same patients that are over three years apart are employed as different samples, incorporating information of test data into the learning process. Rieke et al. [19] trained a 3D CNN for AD classification accuracy. At the end of their visualization efforts, they showed that the model focuses on the medial temporal lobe. Yang et al. [34] also provided visual explanations regarding the $\mathrm{AD}$ from Deep 3D CNNs. They utilized 3D-VGGNet together with 3DResNet. Finally, in 2019, Oh et al. [17] develop a volumetric CNN-based approach for the AD classification task.

It should be noted that the classification performances of these studies are hard to compare as they have trained and tested the models with different sets of participants. The studies also differ in terms of the pre-processing stages, hyperparameter selection, cross-validation $(\mathrm{CV})$ procedure, and evaluation metrics.

\section{METHODS}

In this section, the main components of our framework are presented. We briefly describe the datasets used in the experiments in Subsection III-A, explain the preprocessing steps of $T_{1}$-weighted MRI data in III-B and finally show architectures of the model in III-C.

\section{A. Datasets}

In this study, we use two primary publicly available datasets on AD and Related Dementia: the Alzheimer's Disease Neuroimaging Initiative (ADNI) dataset [35] and Open Access Series of Imaging Studies (OASIS) [36]. These datasets are described in detail below. The characteristics of the subjects included in this study are given in Table I. 
TABLE I: Demographic information of subjects from ADNI and OASIS datasets

\begin{tabular}{lllll}
\hline Dataset & Diagnosis & No. & Sex & Age (mean \pm SD) \\
\hline ADNI & AD & 100 & $44 \mathrm{~F}, 56 \mathrm{M}$ & $74.3 \pm 7.9$ \\
& HC & 100 & $52 \mathrm{~F}, 48 \mathrm{M}$ & $75.0 \pm, 7.1$ \\
\hline OASIS & AD & 100 & $59 \mathrm{~F}, 41 \mathrm{M}$ & $76.7 \pm 7.1$ \\
& HC & 100 & $73 \mathrm{~F}, 27 \mathrm{M}$ & $75.5 \pm 9.1$
\end{tabular}

1) $A D N I: \mathrm{ADNI}^{1}$ is a research initiative that brings together researchers to collect, validate, and utilize several types of data such as clinical, genetic, MRI, PET, and biospecimen to validate biomarkers for AD [35]. ADNI is formed in 2004 and launched three different phases so far, namely ADNI 1, ADNI GO/2, and now ADNI 3. In addition to the first phase, ADNI 2 contains information from 150 elderly controls, $100 \mathrm{EMCI}$ subjects, 150 late mild cognitive impairment (LMCI) subjects, and 150 mild $\mathrm{AD}$ patients.

In this work, we used a subset of ADNI 2 dataset with 200 structural $T_{1}$-weighted MRI scans. From ADNI 2 dataset, we randomly picked 200 subjects, 100 of whom were chosen from the $\mathrm{AD}$ group (44 women and 56 men, age $74.28 \pm 7.96$ years, mean $\pm \mathrm{SD}$ ), while the other 100 from the HC group (52 women and 48 men, age $75.04 \pm 7.11$ years, mean \pm SD). Only the first scan of each patient has been added to the dataset. Patients with a clinical dementia rating (CDR) score of 0 are labeled as HC subjects, whereas the ones whose CDR rating higher than 0 are considered as AD subjects. MPRAGE $T_{1}$ weighted MRI images have been acquired using $3 \mathrm{~T}$ scanners, and consisted of $176 \times 240 \times 256$ (Siemens) and $170 \times 256$ $\times 256$ (Philips) voxels with a size of approximately $1 \mathrm{~mm} \times$ $1 \mathrm{~mm} \times 1.2 \mathrm{~mm}$.

2) OASIS: OASIS ${ }^{2}$ is a project that is intended to promote future discoveries in $\mathrm{AD}$ by providing neuroimaging datasets freely to the scientific community. The project released data in three different phases: OASIS 1-Cross-sectional, OASIS 2Longitudinal, and OASIS-3-Longitudinal. OASIS 1 includes overall 416 subjects ( $316 \mathrm{HC}$ and $100 \mathrm{AD}$ ) aged 18 to 96.

For our experiments, $T_{1}$-weighted MRI scans of 100 healthy subjects [ 73 women and 27 men, age $75.5 \pm 9.1$ years, mean \pm standard deviation (SD)] and $100 \mathrm{AD}$ patients (59 women and 41 men, age $76.7 \pm 7.1$ years, mean \pm SD) have been selected to create a subset of OASIS-1 dataset. Again, the CDR score was 0 for the $\mathrm{HC}$ subjects, 0.5 (very mild), 1 (mild), 2 (moderate), and 3 (severe) were for the AD subjects. MPRAGE $T_{1}$-weighted MRI images have been acquired using a $1.5 \mathrm{~T}$ Siemens scanner. They are in the size of $256 \times 256$ $\times 128$ with voxel size $1 \mathrm{~mm} \times 1 \mathrm{~mm} \times 1.25 \mathrm{~mm}$.

\section{B. Data pre-processing}

Even though CNN models do not require any preprocessing beforehand, an accurate image preprocessing stage could be key to increase the effectiveness of learning and help to

\footnotetext{
${ }^{1}$ The details regarding acquisition protocols can be found at http://adni.loni. usc.edu/methods/documents/mri-protocols/.

${ }^{2}$ More details about the OASIS-1 data can be found at https://www. oasis-brains.org/files/oasiscross-sectionalfacts.pdf.
}

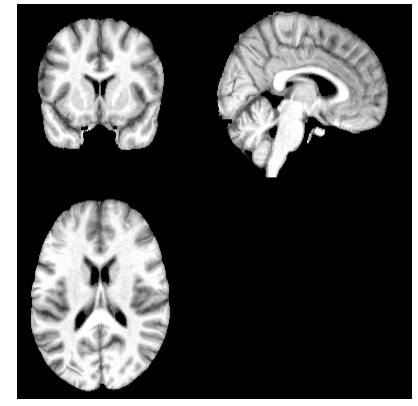

(a) A sample $T_{1}$-weighted MRI slices of a Alzheimer's disease (AD) patient from ADNI dataset after preprocessing - in coronal, sagittal, and axial view (left, right and bottom respectively). (b) A sample $T_{1}$-weighted MRI slices of a Alzheimer's disease (AD) patient from OASIS dataset after preprocessing - in coronal, sagittal, and axial view (left, right and bottom respectively)

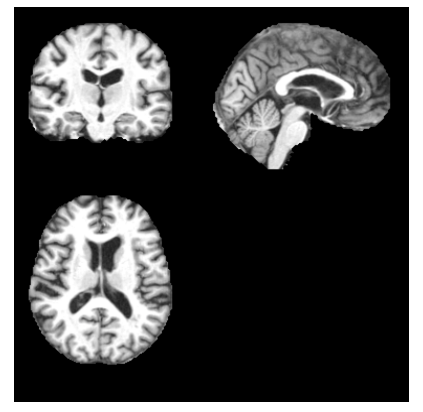

Fig. 2: Example of six Magnetic resonance imaging (MRI) slices of two Alzheimer's Disease (AD) subjects from ADNI and OASIS databases [35], [36].

achieve a good classification performance, particularly in the domain of MRI [37], [38]. We transformed all the data into a standardized structure by performing co-registration with a standard template and skull stripping.

For ADNI, each $T_{1}$-weighted image has been co-registered with the SyN method using standard $T_{1}$-weighted template MNI152 at $1 \mathrm{~mm}$ [39]. After co-registration, the brain mask of the standard space has been applied to each volume to remove extracranial tissues. The final size of the ADNI $T_{1}$-weighted MRI volumes is 182 × 218 x 182 with $1 \mathrm{~mm} \times 1 \mathrm{~mm} \times 1 \mathrm{~mm}$ voxel size.

When it comes to the OASIS dataset, we used the data which was already gain-field corrected. An additional brain masking and re-sampling operations are performed. The final dimension of the $3 \mathrm{D}$ volume is $176 \times 208 \times 176$ with $1 \mathrm{~mm}$ x $1 \mathrm{~mm} \times 1 \mathrm{~mm}$ voxel size [40]. The sample MRI slices from ADNI and OASIS datasets after the pre-processing stage can be seen in Figure 2.

\section{CNN Models: 3D Convolutional Networks}

We created a 3D CNN model inspired by VGG-16 architecture. The model has four convolutional blocks, among which the first two contain two convolutional layers each, and the latter two have three convolutional layers followed by a pooling layer with filter size $2 \times 2 \times 2$. The overview of the $3 \mathrm{D}$ $\mathrm{CNN}$ architecture is shown in Figure 3. A convolutional and a pooling layer has several feature maps, and in most cases, the number of feature maps increases as layers grow. The calculation of the $j^{t h}$ feature map is given by:

$$
y^{j}=f\left(W_{j} * x+b_{j}\right)
$$

where $y^{j}$ be the $3 \mathrm{D}$ array of the $j^{\text {th }}$ feature map in a hidden layer, $\mathrm{x}$ be the 3D array of the input, $b^{j}$ be the scalar bias and $W^{j}$ be the 3D filter with a size of $w \times h \times d . f$ corresponds to an activation function, and $*$ stands for the convolution operation. 


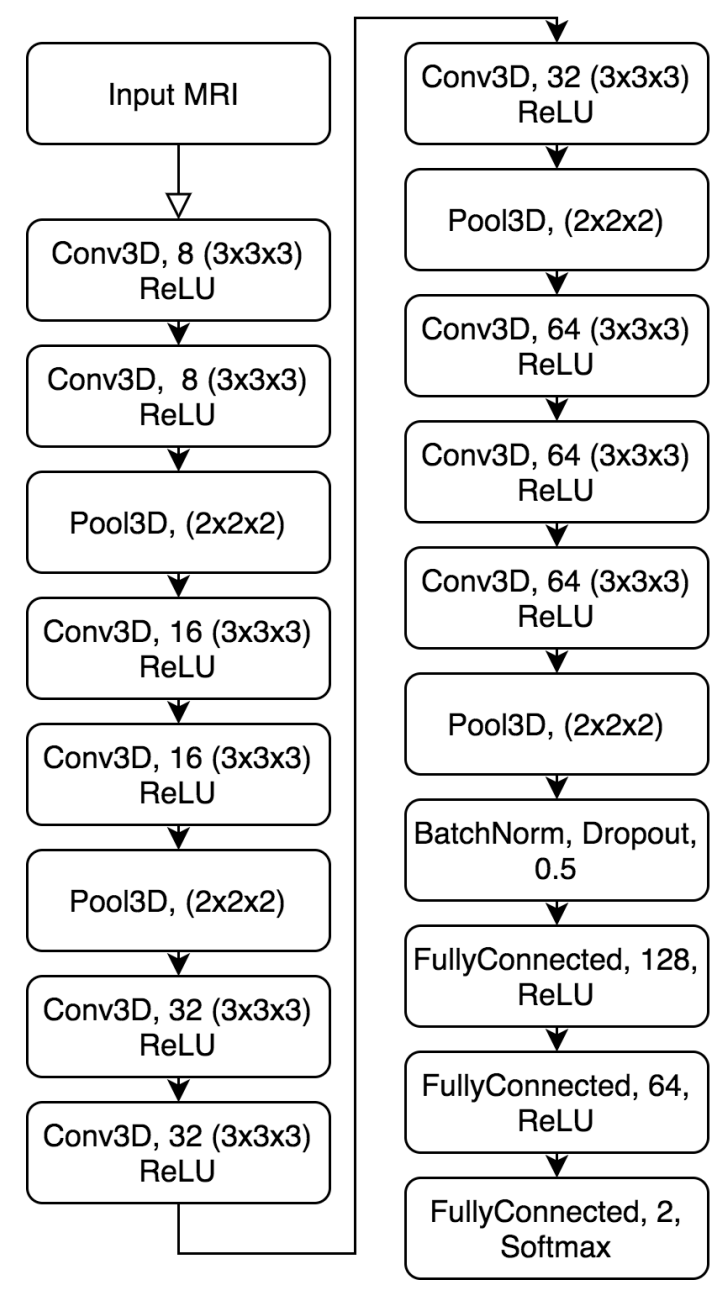

Fig. 3: The architecture of the convolutional neural network (CNN) model used in our AD classification tasks.

The convolution operation $\left[W_{j} * x\right](m, p, q)$, is represented as follows:

$$
\sum_{u=0}^{w-1} \sum_{v=0}^{h-1} \sum_{k=0}^{d-1} W_{j}(w-u, h-v, d-w) x(m+u, p+v, q+k)
$$

After the convolutional blocks, a dropout layer with a probability of 0.5 is applied to avoid overfitting. It is followed by three fully connected layers with 128, 64, and 2 neurons, respectively. The last fully-connected layer with softmax activation provides the output label. The model has been trained with categorical cross-entropy loss and the Adam optimizer with a learning rate of 0.0001 and a batch size of 2 for 200 epochs. Binary cross-entropy loss is computed as:

$$
L(y, p)=-(y \log p+(1-y) \log (1-p))
$$

where $\mathrm{y}$ is the actual label and $\mathrm{p}$ is the predicted label.

Training and validation of our proposed models were performed on a NVidia RTX2080 GPU.

\section{EXPERIMENTAL RESULTS}

The model has been evaluated using five-fold CV. The average accuracy is obtained by repeating 5 times the full 5 -fold cross-validation starting from five different splits of the data into folds. The architecture was built using Keras with TensorFlow backend [41], [42].

The model was tested on two different test sets, each of which contains 40 subjects. Using 5-fold CV, the model achieves $73.4 \% \pm 0.04$ (mean, standard deviation) on ADNI dataset and 69.9\% 0.06 (mean, standard deviation) classification accuracy on the OASIS dataset. The results are comparable to other studies that use different convolutional models for $\mathrm{AD}$ vs. HC classification. In addition, the dataset is divided by subjects, and only one screening of a patient is included in the dataset in order to prevent possible data leakage. For instance, Rieke et al. [19] reported 78\% \pm 0.04 classification accuracy with a similar architecture using ADNI 1 datasets, which contains MRI scans of the subjects up to three-time points (screening, 12 and 24 months; sometimes multiple scans per visit). Following such procedure may cause the scans of the same subject to be in both testing and training set, which could affect the model performance.

\section{CONCLUSION}

In this work, we presented a deep 3D CNN model for the classification of $\mathrm{AD}$ patients using structural brain MRI. We demonstrated the model performance on two primary $\mathrm{AD}$ datasets, namely ADNI and OASIS. Without any feature extraction stage, the model managed to achieve $73.4 \% \pm 0.04$ (mean, standard deviation) and 69.9\% 0.06 accuracy for classification of AD subjects from $\mathrm{HC}$ on ADNI and OASIS datasets respectively. In our future work, we would like to expand this study and archive better classification accuracy through optimizing the network shape and hyperparameters. Moreover, we will use explainable AI techniques to investigate which brain regions or patterns are most important to the model and shed light on the rationale behind the model's predictions.

\section{ACKNOWLEDGMENT}

Data collection and sharing for this project was funded by the Alzheimer's Disease Neuroimaging Initiative (ADNI) (National Institutes of Health Grant U01 AG024904) and DOD ADNI (Department of Defense award number W81XWH-122-0012). ADNI is funded by the National Institute on Aging, the National Institute of Biomedical Imaging and Bioengineering, and through generous contributions from the following: AbbVie, Alzheimer's Association; Alzheimer's Drug Discovery Foundation; Araclon Biotech; BioClinica, Inc.; Biogen; Bristol-Myers Squibb Company; CereSpir, Inc.; Cogstate; Eisai Inc.; Elan Pharmaceuticals, Inc.; Eli Lilly and Company; EuroImmun; F. Hoffmann-La Roche Ltd and its affiliated company Genentech, Inc.; Fujirebio; GE Healthcare; IXICO Ltd.; Janssen Alzheimer Immunotherapy Research Development, LLC.; Johnson Johnson Pharmaceutical Research Development LLC.; Lumosity; Lundbeck; Merck Co., Inc.; 
Meso Scale Diagnostics, LLC.; NeuroRx Research; Neurotrack Technologies; Novartis Pharmaceuticals Corporation; Pfizer Inc.; Piramal Imaging; Servier; Takeda Pharmaceutical Company; and Transition Therapeutics. The Canadian Institutes of Health Research is providing funds to support ADNI clinical sites in Canada. Private sector contributions are facilitated by the Foundation for the National Institutes of Health (www.fnih.org). The grantee organization is the Northern California Institute for Research and Education, and the study is coordinated by the Alzheimer's Therapeutic Research Institute at the University of Southern California. ADNI data are disseminated by the Laboratory for Neuro Imaging at the University of Southern California.

OASIS data were provided by OASIS: Cross-Sectional: Principal Investigators: D. Marcus, R, Buckner, J, Csernansky J. Morris; P50 AG05681, P01 AG03991, P01 AG026276, R01 AG021910, P20 MH071616, U24 RR021382.

\section{REFERENCES}

[1] S. Hague, S. Klaffke, and O. Bandmann, "Neurodegenerative disorders: Parkinson's disease and huntington's disease," Journal of Neurology, Neurosurgery \& Psychiatry, vol. 76, no. 8, pp. 1058-1063, 2005.

[2] H. Braak and E. Braak, "Neuropathological stageing of alzheimer-related changes," Acta neuropathologica, vol. 82, no. 4, pp. 239-259, 1991.

[3] M. D. Weingarten, A. H. Lockwood, S.-Y. Hwo, and M. W. Kirschner, "A protein factor essential for microtubule assembly," Proceedings of the National Academy of Sciences, vol. 72, no. 5, pp. 1858-1862, 1975.

[4] I. Grundke-Iqbal, K. Iqbal, Y.-C. Tung, M. Quinlan, H. M. Wisniewski, and L. I. Binder, "Abnormal phosphorylation of the microtubuleassociated protein tau (tau) in alzheimer cytoskeletal pathology," Proceedings of the National Academy of Sciences, vol. 83, no. 13, pp. 49134917, 1986.

[5] V. L. Villemagne, S. Burnham, P. Bourgeat, B. Brown, K. A. Ellis, O. Salvado, C. Szoeke, S. L. Macaulay, R. Martins, P. Maruff, et al., "Amyloid $\beta$ deposition, neurodegeneration, and cognitive decline in sporadic alzheimer's disease: a prospective cohort study," The Lancet Neurology, vol. 12, no. 4, pp. 357-367, 2013.

[6] E. M. Reiman, Y. T. Quiroz, A. S. Fleisher, K. Chen, C. Velez-Pardo, M. Jimenez-Del-Rio, A. M. Fagan, A. R. Shah, S. Alvarez, A. Arbelaez, et al., "Brain imaging and fluid biomarker analysis in young adults at genetic risk for autosomal dominant alzheimer's disease in the presenilin 1 e280a kindred: a case-control study," The Lancet Neurology, vol. 11, no. 12 , pp. 1048-1056, 2012.

[7] C. R. Jack Jr, V. J. Lowe, S. D. Weigand, H. J. Wiste, M. L. Senjem, D. S. Knopman, M. M. Shiung, J. L. Gunter, B. F. Boeve, B. J. Kemp, et al., "Serial pib and mri in normal, mild cognitive impairment and alzheimer's disease: implications for sequence of pathological events in alzheimer's disease," Brain, vol. 132, no. 5, pp. 1355-1365, 2009.

[8] R. J. Bateman, C. Xiong, T. L. Benzinger, A. M. Fagan, A. Goate, N. C. Fox, D. S. Marcus, N. J. Cairns, X. Xie, T. M. Blazey, et al., "Clinical and biomarker changes in dominantly inherited alzheimer's disease," $N$ Engl J Med, vol. 367, pp. 795-804, 2012.

[9] H. Braak, D. R. Thal, E. Ghebremedhin, and K. Del Tredici, "Stages of the pathologic process in alzheimer disease: age categories from 1 to 100 years," Journal of Neuropathology \& Experimental Neurology, vol. 70, no. 11, pp. 960-969, 2011.

[10] S. Lehericy, M. Baulac, J. Chiras, L. Pierot, N. Martin, B. Pillon, B. Deweer, B. Dubois, and C. Marsault, "Amygdalohippocampal mr volume measurements in the early stages of alzheimer disease.," American Journal of Neuroradiology, vol. 15, no. 5, pp. 929-937, 1994.

[11] M. Bobinski, M. De Leon, J. Wegiel, S. Desanti, A. Convit, L. Saint Louis, H. Rusinek, and H. Wisniewski, "The histological validation of post mortem magnetic resonance imaging-determined hippocampal volume in alzheimer's disease," Neuroscience, vol. 95, no. 3, pp. 721-725, 1999
[12] J. Mortimer, K. Gosche, K. Riley, W. Markesbery, and D. Snowdon, "Delayed recall, hippocampal volume and alzheimer neuropathology: findings from the nun study," Neurology, vol. 62, no. 3, pp. 428-432, 2004.

[13] S. Alam, G.-R. Kwon, J.-I. Kim, and C.-S. Park, "Twin svm-based classification of alzheimer's disease using complex dual-tree wavelet principal coefficients and lda," Journal of healthcare engineering, vol. 2017, 2017.

[14] X. Liu, D. Tosun, M. W. Weiner, N. Schuff, A. D. N. Initiative, et al., "Locally linear embedding (lle) for mri based alzheimer's disease classification," Neuroimage, vol. 83, pp. 148-157, 2013.

[15] K. R. Gray, P. Aljabar, R. A. Heckemann, A. Hammers, D. Rueckert, A. D. N. Initiative, et al., "Random forest-based similarity measures for multi-modal classification of alzheimer's disease," NeuroImage, vol. 65, pp. 167-175, 2013.

[16] Y. Huang, J. Xu, Y. Zhou, T. Tong, X. Zhuang, A. D. N. I. (ADNI, et al., "Diagnosis of alzheimer's disease via multi-modality $3 \mathrm{~d}$ convolutional neural network," Frontiers in Neuroscience, vol. 13, p. 509, 2019.

[17] K. Oh, Y.-C. Chung, K. W. Kim, W.-S. Kim, and I.-S. Oh, "Classification and visualization of alzheimer's disease using volumetric convolutional neural network and transfer learning," Scientific Reports, vol. 9, no. 1, pp. 1-16, 2019.

[18] A. Payan and G. Montana, "Predicting alzheimer's disease: a neuroimaging study with $3 \mathrm{~d}$ convolutional neural networks," arXiv preprint arXiv:1502.02506, 2015.

[19] J. Rieke, F. Eitel, M. Weygandt, J.-D. Haynes, and K. Ritter, "Visualizing convolutional networks for mri-based diagnosis of alzheimer's disease," in Understanding and Interpreting Machine Learning in Medical Image Computing Applications, pp. 24-31, Springer, 2018.

[20] S. Korolev, A. Safiullin, M. Belyaev, and Y. Dodonova, "Residual and plain convolutional neural networks for 3d brain mri classification," in 2017 IEEE 14th International Symposium on Biomedical Imaging (ISBI 2017), pp. 835-838, IEEE, 2017.

[21] J. Wen, E. Thibeau-Sutre, J. Samper-Gonzalez, A. Routier, S. Bottani, S. Durrleman, N. Burgos, and O. Colliot, "Convolutional neural networks for classification of alzheimer's disease: Overview and reproducible evaluation," arXiv preprint arXiv:1904.07773, 2019.

[22] A. Farooq, S. Anwar, M. Awais, and S. Rehman, "A deep cnn based multi-class classification of alzheimer's disease using mri," in 2017 IEEE International Conference on Imaging systems and techniques (IST), pp. 1-6, IEEE, 2017.

[23] K. Gunawardena, R. Rajapakse, and N. Kodikara, "Applying convolutional neural networks for pre-detection of alzheimer's disease from structural mri data," in 2017 24th International Conference on Mechatronics and Machine Vision in Practice (M2VIP), pp. 1-7, IEEE, 2017.

[24] M. Hon and N. M. Khan, "Towards alzheimer's disease classification through transfer learning," in 2017 IEEE International conference on bioinformatics and biomedicine (BIBM), pp. 1166-1169, IEEE, 2017.

[25] J. Islam and Y. Zhang, "Brain mri analysis for alzheimer's disease diagnosis using an ensemble system of deep convolutional neural networks," Brain informatics, vol. 5, no. 2, p. 2, 2018.

[26] A. Valliani and A. Soni, "Deep residual nets for improved alzheimer's diagnosis," in Proceedings of the 8th ACM International Conference on Bioinformatics, Computational Biology, and Health Informatics, pp. 615-615, 2017.

[27] S.-H. Wang, P. Phillips, Y. Sui, B. Liu, M. Yang, and H. Cheng, "Classification of alzheimer's disease based on eight-layer convolutional neural network with leaky rectified linear unit and max pooling," Journal of medical systems, vol. 42, no. 5, p. 85, 2018.

[28] E. Yagis, A. G. S. De Herrera, and L. Citi, "Generalization performance of deep learning models in neurodegenerative disease classification," in 2019 IEEE International Conference on Bioinformatics and Biomedicine (BIBM), pp. 1692-1698, IEEE, 2019.

[29] S. Sarraf and G. Tofighi, "Classification of alzheimer's disease using fmri data and deep learning convolutional neural networks," arXiv preprint arXiv:1603.08631, 2016.

[30] R. Jain, N. Jain, A. Aggarwal, and D. J. Hemanth, "Convolutional neural network based alzheimer's disease classification from magnetic resonance brain images," Cognitive Systems Research, vol. 57, pp. 147159,2019

[31] Y. R. Fung, Z. Guan, R. Kumar, J. Y. Wu, and M. Fiterau, "Alzheimer's disease brain mri classification: Challenges and insights," arXiv preprint arXiv:1906.04231, 2019. 
[32] E. Hosseini-Asl, G. Gimel'farb, and A. El-Baz, "Alzheimer's disease diagnostics by a deeply supervised adaptable $3 \mathrm{~d}$ convolutional network," arXiv preprint arXiv:1607.00556, 2016.

[33] S. Wang, H. Wang, Y. Shen, and X. Wang, "Automatic recognition of mild cognitive impairment and alzheimers disease using ensemble based 3d densely connected convolutional networks," in 2018 17th IEEE International Conference on Machine Learning and Applications (ICMLA), pp. 517-523, IEEE, 2018.

[34] C. Yang, A. Rangarajan, and S. Ranka, "Visual explanations from deep 3d convolutional neural networks for alzheimer's disease classification," in AMIA Annual Symposium Proceedings, vol. 2018, p. 1571, American Medical Informatics Association, 2018.

[35] R. C. Petersen, P. Aisen, L. A. Beckett, M. Donohue, A. Gamst, D. J. Harvey, C. Jack, W. Jagust, L. Shaw, A. Toga, et al., "Alzheimer's disease neuroimaging initiative (adni): clinical characterization," Neurology, vol. 74, no. 3, pp. 201-209, 2010.

[36] D. S. Marcus, T. H. Wang, J. Parker, J. G. Csernansky, J. C. Morris, and R. L. Buckner, "Open access series of imaging studies (oasis): crosssectional mri data in young, middle aged, nondemented, and demented older adults," Journal of cognitive neuroscience, vol. 19, no. 9, pp. 14981507, 2007

[37] R. Cuingnet, E. Gerardin, J. Tessieras, G. Auzias, S. Lehéricy, M.O. Habert, M. Chupin, H. Benali, O. Colliot, A. D. N. Initiative, et al., "Automatic classification of patients with alzheimer's disease from structural mri: a comparison of ten methods using the adni database," neuroimage, vol. 56, no. 2, pp. 766-781, 2011.

[38] D. Lu and Q. Weng, "A survey of image classification methods and techniques for improving classification performance," International journal of Remote sensing, vol. 28, no. 5, pp. 823-870, 2007.

[39] B. B. Avants, N. J. Tustison, G. Song, P. A. Cook, A. Klein, and J. C. Gee, "A reproducible evaluation of ants similarity metric performance in brain image registration," Neuroimage, vol. 54, no. 3, pp. 2033-2044, 2011.

[40] X. Han, R. Kwitt, S. Aylward, S. Bakas, B. Menze, A. Asturias, P. Vespa, J. Van Horn, and M. Niethammer, "Brain extraction from normal and pathological images: a joint pca/image-reconstruction approach," NeuroImage, vol. 176, pp. 431-445, 2018.

[41] F. Chollet et al., "keras," 2015.

[42] M. Abadi, P. Barham, J. Chen, Z. Chen, A. Davis, J. Dean, M. Devin, S. Ghemawat, G. Irving, M. Isard, et al., "Tensorflow: A system for large-scale machine learning," in 12th $\{$ USENIX $\}$ Symposium on Operating Systems Design and Implementation (\{OSDI\} 16), pp. 265283,2016 